\title{
Image de-hazing Method Based on Dark Channel Prior
}

\author{
Xiao Yiran ${ }^{1, a}$, Tian Xiaolin ${ }^{1,2, b}$ \\ ${ }^{1}$ Faculty of Information Technology, Macau University of Science and Technology, Macau, China \\ ${ }^{2}$ Space Science Institute, Macau University of Science and Technology, Macau, China \\ a1753071742@qq.com, bxltian@must.edu.mo
}

Keywords: Dark-channel Prior, Atmospheric Model, Image de-hazing

\begin{abstract}
In this paper, an image de-hazing method based on dark channel prior is discussed. Based on the atmospheric model and physical theory, an improved KPCA method will be used to optimize transmission function instead of filter method before. Meanwhile, a progressive traversal method will be used for estimation of atmospheric light. Also, color domain correction will be added after obtaining the restored image for a better consistency with human visual properties. For different types of fog, such as water-fog in Macau, the method can show a great result.
\end{abstract}

\section{Introduction}

Image dehazing is a popular technology which had made great progress these years. Since 2008, $\operatorname{Tan}^{[1]}$ and Fatla ${ }^{[2]}$, respectively, proposed a dehazing algorithm based on thepriori hypothesis, this idea has become the research hotspots in dehazing area. So far, a large number of researchers have proposed a variety of image dehazing algorithms based on priori hypotheses. The most famous one is image dehazing method based on dark channel prior proposed by $\mathrm{He}$ in 2009. Although the principle is simple, the method can accurately estimate the transmittance intensity of different regions in the fog image and obtain a good dehazing effect, which was awarded as the best paper of CVPR in 2009. Nowadays, the algorithm is used in many dehazing fields, but the results can be dissimilar with different range of transmittance values.

KPCA is a complete mathematical theory, while the most important part in dark-channel prior is a physical model named Atmospheric Scattering Model. The combination of mathematical model and physical model is expected to show a better result in image enhancement.

\section{Proposed dark-channel Method}

We use dark-channel to deal with dehazing problems first. When the weather is foggy and hazy, on the one hand, object surface's reflection light will be attenuated when arriving in collecting device. On the other hand, atmospheric optical and other objects' reflection light will get into the equipment and involved in imaging, and the interference degree increased exponentially with the increase of distance. We have an equation:

Atmospheric Scattering Model $=$

Reflected Light Recession Model + Atmospheric Optical Imaging Model

Reflected light on the surface of the object is reflected by the particles in the air, which causes some light energy loss and spreads to other direction. The light intensity of the acquisition device is reduced, and the image resolution is reduced. When the concentration of the particles in the air is bigger, the distance of the propagation of the light is farther, the stronger the attenuation effect is. 
As shown in Fig.1, firstly we separately extract a beam of light, and $\mathrm{d}$ is a very short distance that the light goes through. The intensity of the light passing through the distance is:

$$
\frac{\mathrm{dE}(\mathrm{x}, \lambda)}{\mathrm{E}(\mathrm{x}, \lambda)}=-\beta(\lambda) \mathrm{dx}
$$

Here, $\beta$ is the atmospheric scattering coefficient. After integration and the assumption that the particles in the air are evenly distributed, we can get:

$$
E(d, \lambda)=E_{0}(\lambda) e^{-\beta(\lambda) d}
$$

This is the result of Reflected Light Recession Model.

When the particle content in the air is higher, the scattering effect makes the air become a " light source ". The farther away from the scene, the more ambient light is collected, and the higher the brightness of the image. We can simplify this model to a mathematical model[3] (Fig.1).

Assuming that the ambient light intensity at infinity is constant $\mathrm{k}$, the luminous flux can be written as:

$$
\operatorname{dI}(x, \lambda)=\operatorname{dVk} \beta(\lambda)=d \omega x^{2} \operatorname{dxk} \beta(\lambda)
$$

According to the rules proposed by Allard in 1876, two formulas can get below:

$$
\begin{aligned}
& \mathrm{dE}(\mathrm{x}, \lambda)=\frac{\mathrm{dI}(\mathrm{x}, \lambda) \mathrm{e}^{-\beta(\lambda) \mathrm{x}}}{\mathrm{x}^{2}} \\
& \mathrm{dL}(\mathrm{x}, \lambda)=\frac{\mathrm{dE}(\mathrm{x}, \lambda)}{\mathrm{d} \omega}=\frac{\mathrm{dI}(\mathrm{x}, \lambda) \mathrm{e}^{-\beta(\lambda) \mathrm{x}}}{\mathrm{d} \omega \mathrm{x}^{2}}
\end{aligned}
$$

After integration and the assumption that $d$ is infinity, we use $\mathrm{Ea}(\mathrm{d}, \lambda)$ to represent the intensity of atmospheric light in a certain distance:

$$
E_{a}(d, \lambda)=E_{\infty}(\lambda)\left(1-e^{-\beta(\lambda) d}\right)
$$

According to the atmospheric scattering model, we can get the final model formula:

$$
E(d, \lambda)=E_{0}(\lambda) e^{-\beta(\lambda) d}+E_{\infty}(\lambda)\left(1-e^{-\beta(\lambda) d}\right)
$$

We simplify the formula for simple calculation ${ }^{[4]}$.

$$
\mathrm{I}(\mathrm{x})=\mathrm{J}(\mathrm{x}) \mathrm{t}(\mathrm{x})+\mathrm{A}(1-\mathrm{t}(\mathrm{x}))
$$

$\mathrm{I}$ is the known haze picture, $\mathrm{J}$ is the original picture which need restoration, while the $\mathrm{t}$ and $\mathrm{A}$ are parameters. Therefore, we change single image dehazing into the calculation of transmissivity $t$ and atmospheric light $\mathrm{A}$.

The dark channel prior is based on the following observation on haze-free outdoor images: in most of the non-sky patches, at least one color channel has very low intensity at some pixels. In other words, the minimum intensity in such a patch should have a very low value[5]. Formally, for an image J, we define:

$$
\mathrm{J}^{\mathrm{dark}}(\mathrm{x})=\min _{\mathrm{c} \in\{\mathrm{r}, \mathrm{g}, \mathrm{b}\}}\left(\min _{\mathrm{y} \in \Omega(\mathrm{x})}\left(\mathrm{J}^{\mathrm{c}}(\mathrm{y})\right)\right)
$$



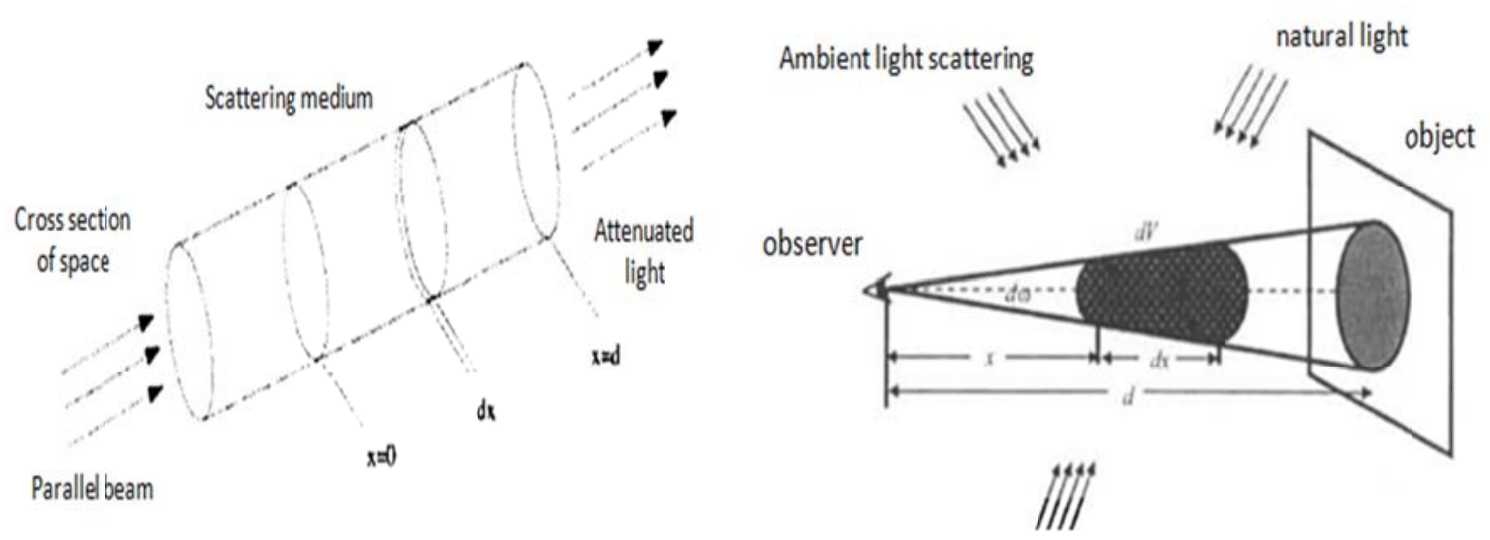

Fig 1. Reflected Light Recession Model and Atmospheric Optical Imaging Model

Our observation says that except for the sky region, the intensity of Jdark is low and tends to be zero, if $\mathrm{J}$ is a haze-free outdoor image. We call Jdark the dark channel of $\mathrm{J}$, and we call the above statistical observation or knowledge the dark channel prior.

Here, we first assume that the atmospheric light $\mathrm{A}$ is given. We further assume that the transmission in a local patch $\Omega(\mathrm{x})$ is constant. We denote the patch's transmission as $\mathrm{t}^{2}(\mathrm{x})$. We have:

$$
\min _{\mathrm{y} \in \Omega(\mathrm{x})}\left(\frac{\mathrm{I}^{\mathrm{c}}(\mathrm{y})}{\mathrm{A}^{\mathrm{c}}}\right)=\tilde{\mathrm{t}}(\mathrm{x}) \min _{\mathrm{y} \in \Omega(\mathrm{x})}\left(\frac{\mathrm{J}^{\mathrm{c}}(\mathrm{y})}{\mathrm{A}^{\mathrm{c}}}\right)+(1-\tilde{\mathrm{t}}(\mathrm{x}))
$$

Then we minimize the three channels, and according to the priori assumption of dark-channel, the minimum value of the channel in the local region approaches 0 , and the atmospheric light $\mathrm{A}$ is always greater than 0 , then we can finally get the formula for transmittance $\tilde{t}(\mathrm{x})$ :

$$
\tilde{\mathrm{t}}(\mathrm{x})=1-\omega \min _{\mathrm{c}}\left(\min _{\mathrm{y} \in \Omega(\mathrm{x})}\left(\frac{\mathrm{I}^{\mathrm{c}}(\mathrm{y})}{\mathrm{A}^{\mathrm{c}}}\right)\right)
$$

The nice property of this modification is that we adaptively keep more haze for the distant objects. The value of $\omega$ is application-based. Normally, the value is 0.95 in most cases.

However, the transmittance image obtained is in a grid shape, not accurate. Thus an optimized operation becomes necessary. A new KPCA method was used here, used Gaussian Radial Basis Function.

$$
\mathrm{k}(\mathrm{X}, \mathrm{Y})=\exp \left(-\mathrm{q}\|\mathrm{X}-\mathrm{Y}\|^{2}\right)
$$

Also, the value of atmospheric light A should be known. A method named progressive traversal was used to estimate the value of A. First of all, traverse the entire image with the $1 / 4$ size of the original image, select the window which is brightest. Second, adjust the window to the $1 / 4$ of the selected area, and then traverse the area. Repeat the work until the selected window size is smaller than the specified threshold value. In the experiment, the selected final window is not larger than the $1 / 400$ of the original image. This method can quickly and effectively deal with atmospheric optical estimation.

After obtaining the transmittance of the optimized image, combined with the estimated value of atmospheric light $\mathrm{A}$, the result can be easily calculated and simulated.

\section{Experimental Results}

Based on the improvement and attempts in KPCA method, a new kernel function was used to 
optimize the transmission function.

Classic fog pictures and Macau fog pictures (with much water in air) are put here, with comparison between the results of dark channel prior and histogram equalization.

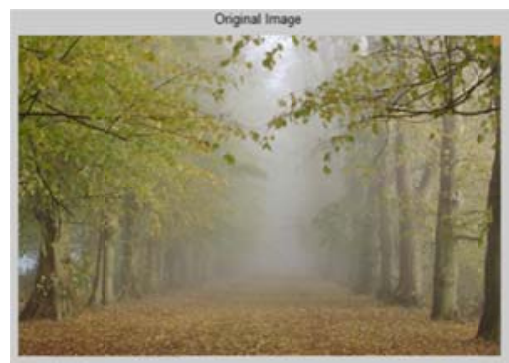

i.original image

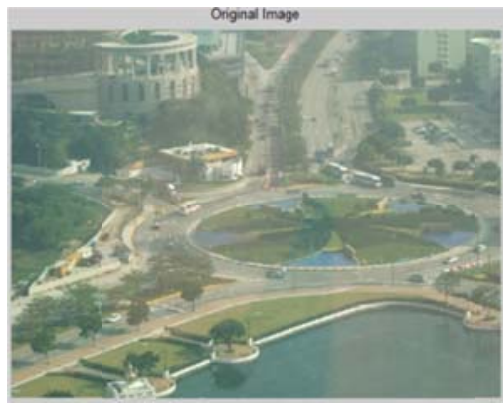

i.original image

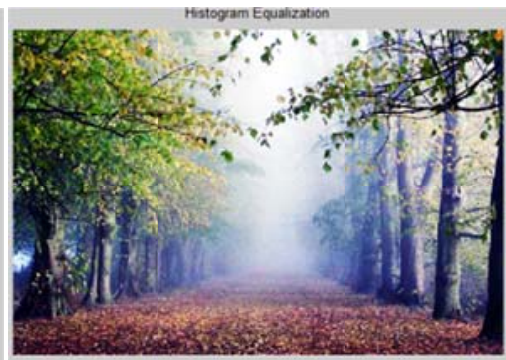

ii. histogram equalization

Fig 2. Classic Fog Pictures

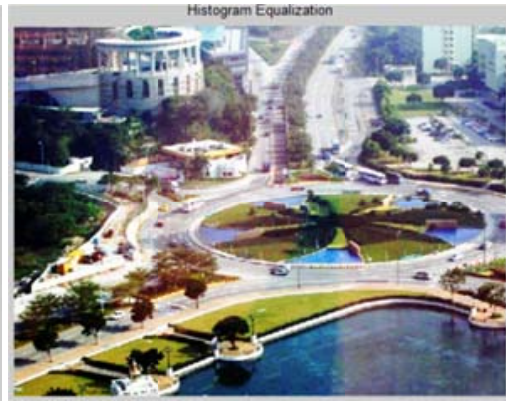

ii. histogram equalization

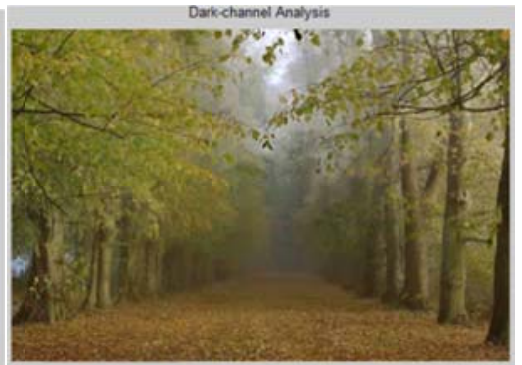

iii.dark-channel analysis

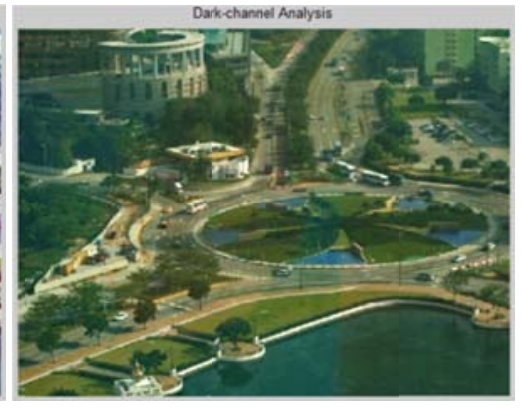

iii.dark-ıchannel analysis

Fig 3. Macau Fog Pictures

\section{Acknowledgements}

This work was financially supported by the Science and Technology Development Fund of Macao (No. 099/2016/A3).

\section{References}

[1] Tan RT.Visbiility in bad weather from a single image[C]//Computer Vision and Pattern Recognition, 2008.CVPR 2008.IEEE Conference on IEEE, 2008: 1-8.

[2] Fattal R.Smgleimage dehazing[C]//ACM Transactions on Graphics(TOG).ACM, 20018,27(3):72.

[3] MA Shiping, ZHANG Ming, BI Duyan, et al. Novel detail preserving Retinex algorithm for image enhancement[J]. Journal of Xidian University, 2010, 37(3): 541-546.

[4] NAN Dong, BI Duyan, XU Yuelei, WANG Shiqiang, LU Xiaolong, Image dehazing method based on dark channel prior, Journal of Central South University (Science and Technology).

[5] ShiWenxuan,WuMinyuan,DengDexiang,et al. Implementation of eliminating cloud and mist noise from remote sensing images[ J]. Optics and Precision Engineering, 2010, 18 ( 1):266-272. 